\section{LEARNING FROM EXCELLENCE IN HEALTHCARE PRACTICE: A POSITIVE EVENT REPORTING SYSTEM}

Mercy Murinye Magwenzi. UK

10.1136/bmjpo-2021-RCPCH. 144

Background Many healthcare institutions have reporting systems in place for when things go wrong as a mechanism to learn from our failures and improve patient safety. However, studies report limitations with this practice. Other studies have shown that nurturing positivity in individuals and teams improves resilience whilst staff morale has been found to have a strong relationship with patient experience.

Objectives The aim was to encourage all staff working in the Department of Paediatrics to recognise and report when things go well, for individuals to reflect on these experiences and for the department to learn from these positive events.

Methods The Positive Event Reporting initiative offers the opportunity for anyone working in the department to nominate any member of staff for excellence in practice using an online form or a paper form. There are no limitations to what may be reported as excellent practice and the initiative is promoted via posters around the department, through social media, email canvassing and individual conversations. Each nominee receives a STAR award, a certificate acknowledging their nomination, with suggested appreciative enquiry questions for their reflection.

A small multidisciplinary team of front-line clinical staff champion the initiative on a volunteer basis, reviewing all reports for themes, creating the award certificates as well as the monthly summaries of learning points and themes which are shared with the whole department through e-newsletters and posters on social media groups.

Results Benefits are experienced in the following areas

- The reported individuals have the opportunity to reflect and learn from their practice and the awards provide evidence of practice for portfolios

- The STAR awards include details of why they were nominated and are kept private to recipients as this has been found to avoid a competitive toxic environment.

- The nominators identify the good practice which they report and help to shift focus towards recognising positive events cultivating a positive culture in the department.

- The whole department learns from these experiences and other staff can model their practice

Staff and management teams have welcomed the initiative. Initial survey results are indicative of increased learning from positive events as well as improved morale among recipients whose excellent practice has been recognised. A steady rise in reports has also been demonstrated over time.

Conclusions Learning from positive events boosts staff morale which in turn can improve the patient experience whilst improving the work culture of a department. Staff have welcomed this initiative which is a positive agent for change and plans are to roll out the initiative to the wider trust. The main administrative challenge is reviewing reports and typing out awards. This is becoming more streamlined through the use of mainly online reporting forms with room for further improvement.

\section{0 IMPACT AND SATISFACTION OF LOCAL PAEDIATRIC ONCOLOGY SERVICES AMONGST PATIENTS AND PARENTS}

Victoria Beraud, Ahsen Razzaq, Mya Aye, Jodie Savage. UK

\subsection{6/bmjpo-2021-RCPCH.145}

Background In many countries, children under the age of 16 are seen in both primary and secondary care. In the UK and Ireland however, there's a network of tertiary specialist centres known as Principal Treatment Centres (PTCs). These are very important as they coordinate and designate the most qualified hospital for children and young people (CYP) with cancer, making sure they are given the best treatment possible.

Each PTCs share care with local hospitals through Paediatric Oncology Shared Care Units (POSCU), enabling children to be supported and treated closer to home. Furthermore, every CYP with cancer are offered a Key Worker, their main point of contact who provides support throughout their convalescence and ensures the appropriate co-ordination of care.

An oncology lead specialist nurse and her team provide different types of services, such as central lines care at home, taking blood, educating schools and reducing any barriers or concerns, to social work like providing support for daily living, giving advice on housing or financial issues.

Objectives Our aim is to demonstrate the beneficial impact of these services on patients and their families by analysing the satisfaction and support provided to them.

Methods Two surveys on patient and parent's satisfaction in paediatric oncology services at local POSCU were conducted, with questionnaires given to all families on active treatment during these periods. The first survey was done in 2016 with $55 \%$ responses and results were compared with two national and regional existing surveys. The second survey was done in 2020 with 54\% responses.

Results The importance of POSCU is evidenced in both surveys. In the 2020 survey, all participants felt a sense of trust/ confidence with the paediatric community nurses/consultants; and in the 2016 survey, all participants believed the care of the CCN was very satisfactory. Both survey results show the significance of a shared care plan and the effect it has on patient satisfaction.

A negative feature of any shared care plan is miscommunication between the PTC and local hospital; however, the survey responses in 2020 showed that no one felt the communication between the local and PTC was below satisfactory (with $71 \%$ definitely satisfied). This was also supported in the 2016 survey where 91\% felt communication between the POSCU and PTC was well/very well. In the 2020 survey a patient explained that 'communications between local \& PTC [...] have been excellent', further emphasising good communication between the two centres. Additionally, POSCU is valued by patients' families, being described in the 2020 survey as 'the best treatment from [...] extraordinary Paediatric Consultant, and from all the Community Nurses'.

Conclusions To conclude, both these surveys showed the beneficial impact of POSCU in the care of CYP with cancer and their families but also the satisfaction of the support brought by these services. This study shows the importance of having these services in place to help reduce these miscommunications and support locally. 
Based on these results, POSCU services should be further analysed and considered in other areas and countries, promising a great beneficial impact on children and their families.

\section{THE OUTCOME OF A SUPERVISED, EXERCISE- PROGRAMME, FOR YOUNG PEOPLE WITH CEREBRAL PALSY: EFFECTS ON PHYSICAL ACTIVITY, FUNCTIONAL AND PARTICIPATION}

Natalie R Walsh, Sarah Rand, Hani F Ayyash, Michael Ogundele. UK

\subsection{6/bmjpo-2021-RCPCH.146}

Background Young-people with Cerebral Palsy (CP) have been shown to have reduced physical fitness estimated at $15-28 \%$ less than healthy age matched children. As a result, they are at an increased risk of developing secondary health consequences due to a reduction in physical activity levels, lower cardiorespiratory capacity, and a decrease in muscle strength. There is limited research investigating the impact of a targeted exercise-programme for these young-people. The aim of this project was to conduct a service evaluation to explore the effects of a community-based, exercise-programme, for young people (age 9-13 years) with CP (GMFCS Levels-I-III).

Objectives

1. To design and implement a community-based exerciseprogramme for young-people with CP.

2. To investigate the impact on physical activity levels using daily step-count data and Physical Activity Questionnaire for Older Children (PAQ-C) scores.

3. To investigate the impact on functional-mobility using TimedUp-and-Go (TUG) times.

4. To investigate participation scores using the Child and Adolescent Scale of Participation (CASP) scores.

Methods Young people with CP were identified using inclusion and exclusion criteria from a single centre. Approvals were granted and consent was obtained from all participants and their parents/carers. A weekly, individualised, physiotherapyled, exercise-programme was completed at a large public leisure centre for six consecutive weeks. Outcome measures were completed to measure changes in physical activity, functionalmobility, and participation at pre-intervention, post-intervention and three-month follow-up.

Results Eight young-people with CP consented and four participants completed the intervention and outcomes. The mean daily step-count increased from post-intervention for three-participants (range -1914.06-2096.86 steps) and was higher on weekdays (range $6410(\mathrm{SD}= \pm 58.35)$ to 664.49 steps $(\mathrm{SD}=$ $\pm 1367.92)$ ) than weekend-days (range $461.35 \quad(\mathrm{SD}= \pm 67.84)$ to 5586.64 steps $(\mathrm{SD}= \pm 539.17))$.

The PAQ-C-scores increased for all participants post-intervention (within-participant-difference range (WPDR) 0.21 $(\mathrm{SD}= \pm 0.38)$ to $0.58(\mathrm{SD}= \pm 0.70))$ and varied at follow-up (WPDR $-0.46(\mathrm{SD}= \pm 0.72)$ to $0.69(\mathrm{SD}= \pm 0.80))$. The TUGtimes improved for two-participants post-intervention (WPDR $-24.29(\mathrm{SD}= \pm 6.13)$ to 1.28 seconds $(\mathrm{SD}= \pm 0.57))$ and was maintained at follow-up (WPDR $-23.70(\mathrm{SD}= \pm 6.22)$ to 1.17 seconds $(\mathrm{SD}= \pm 0.42))$. The CASP-scores improved for threeparticipants (range 2 to 11 ) and was maintained at follow-up (2 to 12 ).

Conclusions The aim of this project was to explore the feasibility and effects of a community-based exercise-group for young-people with CP. Overall the implementation and integration of the programme into the gym-environment was very successful. Attendance levels were $90 \%$ and all participants reported excellent subjective levels of enjoyment. The results of this project demonstrate preliminary, positive evidence for the effectiveness of the intervention on physical activity, functional-mobility, and participation measures in this small sample. The sample size and data collection were unfortunately affected by the Covid-19 pandemic. Therefore, additional data collection will be conducted in the future to further investigate the impact of the programme. It is clear that further studies are required to investigate outcome measures, to record the effects of interventions on physical activity, functional-mobility, and participation in young-people with CP. This preliminary study shows promise for the positive impact of community-based, exercise-programmes and should be considered as an additional option to facilitate young-people with $\mathrm{CP}$ to increase their participation, and to incorporate physical activity into their daily lives.

\section{EFFECT OF COVID-19 LOCKDOWN ON THE CONFIGURATION AND IMPLEMENTATION OF SERVICES FOR CHILDREN AND YOUNG PEOPLE WITH NEURODEVELOPMENTAL DISORDERS IN THE UK}

Michael Ogundele, Hani Ayyash. UK

\subsection{6/bmjpo-2021-RCPCH.147}

Background The pandemic corona virus outbreak is having an impact on the lives of every member of the society with lockdown measures to limit the virus spreading through Schools shutting down, limited contact with family and friends, as well as social and leisure activities severely curtailed. This also necessitated unavoidable changes to various healthcare services provision worldwide.

Emotional, behavioural, Neurodevelopmental and Intellectual disorders (EBNDID) including ADHD, Autism, Epilepsy and Cerebral palsy affect up to $15 \%$ of the childhood population.

Objectives We aimed to identify pragmatic changes made to services provision for Children and Young people (CYP) with EBNDIDs and help disseminate examples of best practice among $\mathrm{CCH}$ Clinicans, while preparing for future pandemic lockdowns.

Methods We carried out a literature review about the impact of COVID-19 pandemic on healthcare services provided for children and adolescent with ADHD from several databases including Pubmed, PMC, CINAHL, Embase, PsycINFO and Ovid, with no limitations in terms of language and date of publication from inception to Dec 2020. This was supplemented by an online scoping survey among selected members of the George-Still Forum (National ADHD Network) run between June and July 2020.

Results 62 responses were obtained from the online $(62 \%$ rate). Several national and international professional bodies provided clinical guidance to clinicians about necessary service modifications to ensure that patients continue to have access to best evidence-based care despite the limitations imposed by the pandemic, but the awareness among frontline Paediatricians was limited. The clinical recommendations have centred around (i) expansion of various forms of remote and online services, (ii) Infection control strategies (iii) and staff redeployment to areas of higher clinical demands. $8 \%$ of the surveyed 